\title{
To hydrate or not to hydrate? Lessons learned from the AMACING trial
}

\author{
Estelle C. Nijssen ${ }^{1} \cdot$ Patty J. Nelemans ${ }^{2} \cdot$ Roger J. Rennenberg ${ }^{3} \cdot$ Vincent van Ommen $^{4} \cdot$ Joachim E. Wildberger $^{1}$
}

Received: 23 March 2019 / Revised: 1 May 2019 / Accepted: 2 June 2019

(c) Springer Nature Singapore Pte Ltd. 2019

The number of diagnostic and interventional procedures with iodinated contrast material is steadily growing. In 2005, it was estimated that over 75 million injections were administered each year worldwide [1]. The benefits of contrast procedures-CT scans for example-are obvious, but injection of the contrast material may also incur some risk.

Iodinated contrast media were first accidentally discovered in the 1920s [2]. Since then they have evolved from extremely toxic, cell-invading salts, to relatively inert, complex benzene molecules [3]. Despite this evolution, intravascular injection of iodinated contrast material may still have systemic and (renal) hemodynamic consequences. The most important of these is contrast-induced nephropathy (CIN), also known as post-contrast acute kidney injury (PC-AKI): an acute decline in renal function, occurring a few days after contrast administration.

The first report of acute renal failure following contrast media injection dates from 1954 [4]. Cases of post-contrast renal function decline have been consistently reported thereafter, some progressing to further renal function decline, dialysis, and death [5-8]. To improve safety, clinical guidelines on the use of intravascular iodinated contrast material have been issued and implemented in hospitals the world over [9-12]. They are generated by dedicated committees and regularly updated. Examples of umbrella organisations issuing such guidelines are the American College of Radiology (the 11th edition of their 'contrast manual' was issued in 2017), the European Society of Urogenital Radiology

Estelle C. Nijssen

estelle.nijssen@mumc.nl

1 Department of Radiology and Nuclear Medicine, Maastricht University Medical Centre, PO Box 5800, 6202 AZ Maastricht, The Netherlands

2 Department of Epidemiology, Maastricht University, 6202 AZ Maastricht, The Netherlands

3 Department of Internal Medicine, Maastricht University Medical Centre, 6202 AZ Maastricht, The Netherlands

4 Department of Cardiology, Maastricht University Medical Centre, 6202 AZ Maastricht, The Netherlands (version 10.0 of their 'guidelines on contrast agents' booklet was released in 2018), and the Royal Australian and New Zealand College of Radiologists (version 2.3 of their 'iodinated contrast guidelines' was issued in 2018) [9-11]. The main recommendation of the guidelines is prophylactic intravenous hydration for the prevention of CIN, before and after contrast administration.

In the Netherlands, clinical guidelines on the use of intravascular iodinated contrast material became part of a nationwide plan which was initiated in 2008 by the Dutch authorities to improve hospital safety [13]. The main recommendations were threefold: (1) determine the renal function (estimated glomerular filtration rate, eGFR) of all patients undergoing a procedure with intravascular iodinated contrast material; (2) identify those patients considered to be at risk of CIN (i.e., patients with eGFR less than $60 \mathrm{~mL} /$ $\mathrm{min} / 1.73 \mathrm{~m}^{2}$ combined with diabetes or $>1$ risk factor [age over 75 years, anaemia, cardiovascular disease or use of nephrotoxic medication], or with eGFR less than $45 \mathrm{~mL} /$ $\min / 1.73 \mathrm{~m}^{2}$ ); (3) administer intravenous normal saline during $4-12 \mathrm{~h}$ before and $4-12 \mathrm{~h}$ after the contrast procedure to patients at risk of CIN.

In the Netherlands, the recommendations were imposed on all centres, and adherence was used as an indicator of hospital quality and safety. Since the prophylaxis requires a minimum of 8-24 h hospitalisation, these recommendations had far reaching consequences for patient, hospital, and health care budgets. On the other hand, evidence of prophylaxis effectiveness was lacking.

Any therapy or medicine represents a balancing act between positive and negative effects. In the case of prophylactic intravenous hydration, neither effect was properly investigated before its introduction as standard care. Although hundreds of trials had been done comparing various prophylactic strategies to each other, and indeed by 2012 some 5,000 papers had been published on the subject, none questioned the "gold standard" of intravenous normal saline. Guideline committees acknowledged that randomised trials comparing prophylactic intravenous hydration to a proper control group without prophylaxis were not available, but 
stated that carrying out such trials would be ethically unacceptable given the current understanding of CIN [14].

At our centre, based on our daily practice, we were not convinced that these extensive guidelines were the best approach. Nor did we agree that such a trial would be unethical: we had already encountered some serious complications after the prophylactic treatment such as symptomatic heart failure, including deaths. Based on these and other deaths deemed avoidable in retrospect, the Commissie Onderzoek Overleden Patienten of Maastricht UMC+ (COOP: a committee which investigates all in-hospital deaths) opened the discussion on burdens of interventions that exceed the coping ability of fragile patients, and sometimes lead to serious complications and death. Taking all the above into consideration, not knowing whether the benefits of prophylactic intravenous hydration outweighed this risk was, to our minds, unacceptable.

We started with a short explorative retrospective analysis of our in-house data [15]. 419 consecutive patients who underwent an elective coronary angiography or intervention were screened. 47 of these met the guideline criteria for high-risk patients. In accordance with the in-house protocol at the time, only 9 of the 47 patients had received prophylactic intravenous fluids, and 38 received none. Despite this, only one in $47(2.1 \%)$ at risk patients developed CIN. This incidence of CIN was much lower than incidences previously reported in literature (ranging from 2 to $50 \%$ or more) [16]. Furthermore, renal function normalised within a few weeks, and no clinically relevant consequences were observed.

This set the stage for the AMACING trial [17]. The aim of the trial was to evaluate the clinical and cost-effectiveness of standard prophylactic hydration according to the guidelines, by comparing patients at risk of CIN who received prophylaxis to those who did not. For the randomised controlled trial, a non-inferiority design was chosen, because although not giving prophylaxis might be expected to increase CIN incidence, it would also reduce patient and hospital burden, and health care costs. Furthermore, although sometimes associated with longer term morbidity and mortality, CIN usually resolves without clinically relevant consequences. Lastly, not giving prophylaxis would mean avoiding complications of intravenous hydration.

For the AMACING trial, we prospectively screened all 28,803 elective procedures requiring iodinated contrast material carried out over a 2-year period at our centre [18]. 1120 patients met the inclusion criteria (i.e., high-risk patients according to the guidelines with eGFR $<60 \mathrm{~mL} /$ $\mathrm{min} / 1.73 \mathrm{~m}^{2}$ combined with risk factors for CIN) $[10,13]$. $59 \%(n=660)$ of eligible patients agreed to participate in the trial, and these were randomised 1:1 to either standard prophylactic hydration according to the guidelines or no prophylaxis. In view of patient safety, we excluded patients with severe chronic kidney disease from the trial, i.e., those with eGFR $<30 \mathrm{~mL} / \mathrm{min} / 1.73 \mathrm{~m}^{2}$. After these exclusions, the included population still represented approximately $90 \%$ of patients to whom the guideline recommendation of standard prophylaxis was applicable.

Primary results showed that not giving prophylaxis was non-inferior to standard prophylaxis with normal saline. CIN incidences were 8/296 (2.7\%) in the standard prophylaxis group versus $8 / 307$ (2.6\%) in the no prophylaxis group (absolute difference in proportions, no prophylaxis group minus prophylaxis group, $-0.10 \%, 95 \% \mathrm{CI}-2.25$ to 2.06 , $p=0.4710$ ). The upper limit of the one-sided $95 \%$ confidence interval excludes an increase in CIN by $2.1 \%$ or more (the non-inferiority margin) in the absence of prophylaxis. No dialysis or related deaths occurred within 1 month. However, $5.5 \%$ of intravenously hydrated patients suffered complications such as heart failure after the prophylactic treatment (13/328 symptomatic heart failure, 4/328 arrhythmia, $1 / 328$ hyponatremia). Finally, medical costs per patient were almost twice as high for prophylaxis patients than for no prophylaxis patients (mean difference approximately $\$ 750$ per patient).

The AMACING data led to the conclusion that, assuming optimal contrast media administration, withholding prophylaxis for patients with eGFR $\geq 30 \mathrm{~mL} / \mathrm{min} / 1.73 \mathrm{~m}^{2}$ might be considered without compromising patient safety.

Secondary outcomes of the trial included incidences of adverse outcomes up to 1-year post-contrast. The 1-year AMACING data showed no clinically relevant differences in 1-year dialysis, 1-year mortality, long-term change in serum creatinine from baseline, or renal events between the no prophylaxis and intravenously hydrated groups [19]. The observed differences between no prophylaxis and prophylaxis groups were consistently small and not significant. This confirmed the earlier conclusion that prophylaxis could safely be withheld for this population.

The AMACING trial prompted an amendment to several guidelines. The guideline committees of the Netherlands (NVvR) and the United Kingdom (NICE) carried out exceptional reviews to incorporate the findings into their guidelines: that routine use of prophylactic intravenous hydration in high-risk patients with eGFR $30-59 \mathrm{ml} / \mathrm{min} / 1.73 \mathrm{~m}^{2}$ may not be beneficial and may inadvertently cause harm through fluid overload [20, 21].

Currently, clinical guidelines have been updated to recommend prophylactic intravenous hydration for patients with eGFR $<30 \mathrm{~mL} / \mathrm{min} / 1.73 \mathrm{~m}^{2}$ only [20-22]. The change is undoubtedly a net improvement, as implementation will mean avoiding unnecessary complications of prophylactic treatment, a considerable reduction in hospital and patient burden, and health care budget savings of \$56-114 million a year in the Netherlands (with 17 million Dutch residents in 2017) [18]. Extrapolating the AMACING results to possible 
consequences worldwide, conservative estimates are that savings for health care budgets are over $\$ 2270,000,000$, that 4 million patients need no longer be hospitalised for prophylaxis, and that over 200,000 patients no longer suffer complications, each year, worldwide. Note that worldwide adherence to the guidelines is assumed in these estimations, which of course is not true. On the other hand, the above estimates are based on the number of intravascular injections carried out worldwide in the year 2005, and the costs are indexed to the year 2015. Assuming a yearly increase of $9 \%$ in the number of injections with iodinated contrast, 75 million injections would now be 250 million injections a year. The above estimations are, therefore, probably not too far off the mark.

The new prophylaxis threshold at eGFR $<30 \mathrm{~mL} /$ $\min / 1.73 \mathrm{~m}^{2}$, however, has not been chosen because of (new) evidence that these patients may profit from prophylactic intravenous hydration. Indeed, very little data and no rigorous randomised trials evaluating prophylaxis are available in literature.

Ideally, the new guidelines would be subjected to a sufficiently powered and robust randomised controlled trial, comparing the recommended prophylaxis to no prophylaxis in the new high-risk population. However, such an endeavour may not be as simple as it sounds.

An analysis of patients with eGFR $<30 \mathrm{~mL} / \mathrm{min} / 1.73 \mathrm{~m}^{2}$ excluded from the AMACING trial showed that these patients represent a different, more vulnerable population to those with eGFR $30-59 \mathrm{~mL} / \mathrm{min} / 1.73 \mathrm{~m}^{2}$ included in the trial [23]. Incidences of post-contrast adverse events were substantially higher in the eGFR $<30 \mathrm{~mL} / \mathrm{min} / 1.73 \mathrm{~m}^{2}$ population compared to those in the AMACING trial population: CIN $13.6 \%$ versus $2.7 \%(p=0.0019)$; 1 month dialysis $0.9 \%$ versus $0.0 \%(p=0.2646)$; and 1 month mortality $9.2 \%$ versus $0.0 \%(p<0.0001)$. Furthermore, more outliers with extreme post-contrast increases in serum creatinine were observed in the eGFR $<30 \mathrm{~mL} / \mathrm{min} / 1.73 \mathrm{~m}^{2}$ population.

eGFR $<30 \mathrm{ml} / \mathrm{min} / 1.73 \mathrm{~m}^{2}$ patients, then, appear to indeed be truly high risk. However, the low prevalence of eGFR $<30 \mathrm{~mL} / \mathrm{min} / 1.73 \mathrm{~m}^{2}(0.4-0.5 \%$ in the general population, and $0.06-0.5 \%$ in the setting of elective iodinated contrast administration), combined with a relatively small non-inferiority margin (only a small increase in CIN would be deemed acceptable), makes randomised trials of sufficient power unrealistic [18, 24-27].

In a second, larger observational study, all 55,474 elective contrast procedures carried out over a 4-year period at our centre were retrospectively screened [28]. The aim was to gain some insight into the positive and negative effects of prophylactic treatment in high-risk patients with eGFR $<30 \mathrm{~mL} / \mathrm{min} / 1.73 \mathrm{~m}^{2}$, by comparing adverse outcomes between patients with and without prophylaxis. This was possible because treating specialists may decide not to give prophylaxis to patients at risk of complications of intravenous hydration, with aortic stenosis or severe chronic heart failure for example. Differences between prophylaxis and no prophylaxis groups were corrected for observed differences in baseline characteristics.

The resulting adjusted odds ratios for risk of CIN, eGFR decline, and dialysis within 1 month were all lower than 1. Though not significant, the point estimates indicate a trend toward a protective effect of intravenous prophylactic fluids over no prophylaxis for post-contrast renal function decline. Adjusted odds ratios for all-cause mortality within 1 month post-contrast were higher than 1 , however, with point estimates indicating a trend toward higher risk of short-term mortality after prophylaxis as compared to no prophylaxis.

Since the study was observational, confounding by indication may partly be responsible for the increased risk of short-term mortality, but complications of the prophylaxis contributed towards the risk. Amongst 281 prophylaxis patients, 18 (6.4\%) complications occurred after prophylaxis: 15 heart failures including 5 deaths, and 3 arrhythmias. Of all 21 deaths in the analysed prophylaxis patients, $24 \%(5 / 21)$ were related to intravenous fluids.

An exploration of differences in baseline characteristics between patients with and without complications suggested that serious complications can be avoided if cardiac function parameters are given extra and individual attention before deciding whether to administer prophylaxis to high-risk patients with eGFR $<30 \mathrm{~mL} / \mathrm{min} / 1.73 \mathrm{~m}^{2}$ [28].

This conclusion has potential consequences for guidelines and daily clinical practice. Physicians from all specialties are confronted with weighing the benefits against the risks of procedures with iodinated contrast material. To date, most focus on protecting renal function; our data indicate that cardiac parameters should also be considered.

The high incidences of post-contrast adverse outcomes in patients with eGFR $<30 \mathrm{~mL} / \mathrm{min} / 1.73 \mathrm{~m}^{2}$ compared to AMACING trial participants indicate that the updated guidelines now better define the 'high-risk of CIN' patient. Prevalence of eGFR $<30 \mathrm{~mL} / \mathrm{min} / 1.73 \mathrm{~m}^{2}$ is low, but we are still talking about at least 375,000 high-risk patients a year worldwide, who are confronted with the risks of contrast administration and prophylaxis.

Prophylaxis may offer some protection against renal function decline, and looks to be without complications for $93.6 \%$ of patients, but serious complications do occur and when they do, they may be fatal. In the absence of randomised controlled trials, which as we have seen may not be readily feasible, many questions remain open on the subject of prophylaxis in patients with eGFR $<30 \mathrm{~mL} / \mathrm{min} / 1.73 \mathrm{~m}^{2}$. We recommend a thorough, individual evaluation, and deliberation on whether to hydrate or not to hydrate in this, truly high-risk, population. 
AMACING taught us that prophylaxis was not beneficial for patients with eGFR $30-59 \mathrm{~mL} / \mathrm{min} / 1.73 \mathrm{~m}^{2}$, and that patients with eGFR $<30 \mathrm{ml} / \mathrm{min} / 1.73 \mathrm{~m}^{2}$ warrant extra and individual attention in this setting. It also taught us that it pays to scrutinise our daily clinical practice, to be wary of quality measures not backed by randomised trial data, and to dare to put expert opinion to the test [29].

\section{References}

1. Christiansen C. X-ray contrast media: an overview. Toxicology. 2005;209:185-7.

2. Osborne ED, Sutherland CG, Scholl AJ, et al. Roentgenography of urinary tract during excretion of sodium iodide. JAMA. 1923;80:368-73.

3. Buschur M, Aspelin P. Contrast Media: history and chemical properties. Interv Cardiol Clin. 2014;3(3):333-9.

4. Bartels ED, Brun GC, Gammeltoft A, et al. Acute anuria following intravenous pyelography in a patient with myelomatosis. Acta Med Scand. 1954;150:297-302.

5. Kidney Disease: Improving Global Outcomes (KDIGO) Acute Kidney Injury Work Group. KDIGO clinical practice guideline for acute kidney injury. Kidney Int Suppl. 2012;2:1-138.

6. LaBounty TM, Shah M, Raman SV, Lin FY, Berman DS, Min JK. Within-hospital and 30-day outcomes in 107994 patients undergoing invasive coronary angiography with different low-osmolar iodinated contrast media. Am J Cardiol. 2012;109:1594-9.

7. Katzberg RW, Newhouse JH. Intravenous contrast medium-induced nephrotoxicity: is the medical risk really as great as we have come to believe? Radiology. 2010;256:21-8.

8. James MT, Samuel SM, Manning MA, et al. Contrast-induced acute kidney injury and risk of adverse clinical outcomes after coronary angiography: a systematic review and meta-analysis. Circ Cardiovasc Interv. 2013;6:37-43.

9. Committee on Drugs and Contrast Media, American College of Radiology (ACR). Manual on contrast media, version 10.3. http:// www.acr.org/quality-safety/resources/contrast-manual Accessed 22 Mar 2019.

10. European Society of Urogenital Radiology. ESUR guidelines on contrast media, version 8.1. http://www.esur.org/guidelines/. Accessed 22 Mar 2019.

11. The Royal Australian and New Zealand College of Radiologists. RANZCR iodinated contrast guidelines. https://www.ranzcr.com/ fellows/clinical-radiology/professional-documents/. Accessed 22 Mar 2019.

12. ASCI CCT and CMR Guideline Working Group (2010) ASCI 2010 contrast media guideline for cardiac imaging: a report of the Asian Society of Cardiovascular Imaging cardiac computed tomography and cardiac magnetic resonance imaging guideline working group. Int J Cardiovasc Imaging. 26:203-212. http://www.asci-heart .org/notice/view.php? code=high\&page $=1 \&$ number $=69 \&$ keyfi eld=\&key. Accessed 22 March 2019.

13. Veligheids Management Systeem. VMS veiligheidsprogramma: voorkomen van nierinsufficiëntie bij intravasculair gebruik van jodiumhoudende contrastmiddelen. September, 2009. http://www. vmszorg.nl/themas/nierinsufficientie. Accessed 22 March 2019.

14. Stacul F, van der Molen AJ, Reimer P, Webb JAW, Thomsen HS, Morcos SK, et al. Contrast induced nephropathy: updated ESUR Contrast Media Safety Committee guidelines. Eur Radiol. 2011;21:2527-41.

15. Vermeeren MAP. namens de CIN-groep MUMC+Veiligheidsregels jagen kosten op. Medisch Contact. 2011;66:2073-5.
16. Thomsen HS, Morcos SK, Barrett BJ. Contrast-induced nephropathy: the wheel has turned 360 degrees. Acta Radiol. 2008;49:464-657.

17. A Maastricht Contrast-Induced Nephropathy Guidelines Study. CIN Prevention Guidelines: Appropriate \& Cost-effective? (AMACING). Clinicaltrials.gov AMACING https://clinicaltrials.gov/ct2/show/ NCT02106234. Accessed 22 Mar 2019.

18. Nijssen EC, Rennenberg RJ, Nelemans PJ, et al. Prophylactic hydration to protect renal function from intravascular iodinated contrast material in patients at high-risk of contrast-induced acute kidney injury (AMACING): a prospective, randomized, phase 3, controlled, open-label, non-inferiority trial. Lancet. 2017;389:1312-22.

19. Nijssen EC, Rennenberg RJ, Nelemans PJ, et al. Prophylactic intravenous hydration to protect renal function from intravascular iodinated contrast material (AMACING): long-term results of a prospective, randomised, controlled trial. EClinicalMedicine. 2018;4-5:109-16.

20. Nederlandse Vereniging voor Radiologie. Guideline safe use of contrast media. https://www.radiologen.nl/kwaliteit/richtlijnen-veili g-gebruik-van-contrastmiddelen. Accessed 22 March 2019.

21. Surveillance report (exceptional review) 2017-Acute kidney injury: prevention, detection and management (2013) NICE guideline CG169 https://www.nice.org.uk/guidance/cg169/resources/surve illance-report-exceptional-review-2017-acute-kidney-injury-preve ntion-detection-and-management-2013-nice-guideline-cg169-46662 60925/chapter/Surveillance-decision?tab=evidence. Accessed 22 March 2019.

22. Van der Molen AJ, Reimer P, Dekers IA, et al. Post-contrast acute kidney injury: Recommendations for updated ESUR Contrast Medium Safety Committee guidelines: Part 2: risk stratification, role of hydration and other prophylactic measures, patients taking metformin and chronic dialysis patients. Eur Radiol. 2018;28(7):2856-69.

23. Nijssen EC, Nelemans PJ, Rennenberg RJ, van Ommen GV, Wildberger JE. Evaluation of safety guidelines on the use of iodinated contrast material conundrum continued. Invest Radiol. 2018;53:616-22.

24. de Jong PE, van der Velde M, Gansevoort RT, et al. Screening for chronic kidney disease: where does Europe go? Clin J Am Soc Nephrol. 2008;3:616-23.

25. Coresh J, Astor BC, Greene T, et al. Prevalence of chronic kidney disease and decreased kidney function in the adult US population: third National Health and Nutrition Examination Survey. Am J Kidney Dis. 2003;41:1-12.

26. Park S, Kim MH, Kang E, et al. Contrast-induced nephropathy after computed tomography in stable CKD patients with proper prophylaxis: 8-year experience of outpatient prophylaxis program. Medicine (Balt). 2016;95:e3560.

27. From AM, Bartholmai BJ, Williams AW, et al. Mortality associated with nephropathy after radiographic contrast exposure. Mayo Clin Proc. 2008;83:1095-100.

28. Nijssen EC, Nelemans PJ, Rennenberg RJ, van Ommen GV, Wildberger JE. Observational study on prophylaxis in high-risk patients with eGFR $<30 \mathrm{~mL} / \mathrm{min} / 1.73 \mathrm{~m}^{2}$ : get the balance right. Investig Radiol. 2019. https://doi.org/10.1097/RLI.0000000000000570 [Epub ahead of print].

29. Mandrola J. Commentary: contrast-induced nephropathy: signal or noise? 2017. http://www.medscape.com/viewarticle/876852. Accessed 22 March 2019.

Publisher's Note Springer Nature remains neutral with regard to jurisdictional claims in published maps and institutional affiliations. 